\title{
Estudio descriptivo de una cohorte de pacientes con COVID-19 hospitalizados en España
}

\author{
Laura Álvarez-Arroyo, ${ }^{1 *}$ Francisco J. Carrera-Hueso, ${ }^{1}$ David El-Qutob, ${ }^{2}$ Montserrat Robustillo-Villarino, ${ }^{3}$ \\ Ana M. Girona-Sanz, ${ }^{4}$ María T. Pin-Godos, ${ }^{5}$ Paula Sánchez-Monzó, ${ }^{6}$ Rafael Martínez-Gonzálbez, \\ Sonia Cepeda-Madrigal ${ }^{\beta}$ y Fernando Martínez-Martínez ${ }^{9}$ \\ ${ }^{1}$ Servicio de Farmacia; ${ }^{2}$ Servicio de Medicina Interna, Unidad de Alergología; ${ }^{3}$ Servicio de Medicina Interna, Departamento de Reumatología; \\ ${ }^{4}$ Servicio Medicina de Interna, Medicina Digestiva; ${ }^{5}$ Servicio de Medicina Interna, Departamento de Nefrología; ${ }^{6}$ Unidad de Neurofisiología; ${ }^{7}$ Unidad \\ de Informática; ${ }^{8}$ Servicio de Medicina Interna, Departamento de Neumología. ${ }^{1-8}$ Hospital Universitario de La Plana, Castellón, España; ${ }^{9}$ Universidad \\ de Granada, Departamento de Farmacia y Tecnología Farmacéutica, Granada, España
}

\section{Resumen}

Antecedentes: Se han publicado varios estudios descriptivos de cohortes de pacientes afectados por COVID-19. Objetivo: Describir las características de pacientes con infección por SARS-CoV-2 que ingresaron al Hospital Universitario de La Plana, Castellón, España. Métodos: Estudio observacional de cohortes retrospectivo, que incluyó pacientes de 18 años o mayores que ingresaron en forma consecutiva con infección confirmada por SARS-CoV2; se describen características demográficas, comorbilidades, síntomas clínicos, resultados de laboratorio y pruebas radiológicas. Resultados: El estudio incluyó 255 pacientes con edad promedio de 70 años; $54.9 \%$ fue del sexo masculino. Las comorbilidades más frecuentes fueron hipertensión arterial (58 \%), dislipemia (42.4\%), diabetes (25.5\%) y obesidad (24.3\%). La mediana de días del inicio de síntomas clínicos antes del ingreso fue de siete. Las manifestaciones más frecuentes previas al ingreso fueron fiebre (74.5\%), tos seca (61.2\%), malestar general (51.8\%) y disnea (51.0\%); 19 pacientes (7.4\%) ingresaron a la unidad de cuidados intensivos, donde la mortalidad fue de $50 \%$; la mortalidad total fue de $16.9 \%$. Conclusiones: Nuestra cohorte refleja características similares a las de otras series europeas. La mortalidad fue inferior a la de estudios similares.

PALABRAS CLAVE: COVID-19. SARS-CoV-2. Neumonía viral. Estudio de cohortes. Coronavirus.

\section{Descriptive study of a cohort of COVID-19 hospitalized patients in Spain}

\section{Abstract}

Background: Several descriptive cohort studies of patients affected by COVID-19 have been published. Objective: To describe the characteristics of patients with SARS-CoV-2 infection who were admitted to Hospital Universitario la Plana, Castellón, Spain. Methods: Retrospective, observational cohort study that included 18-year-old or older patients who were consecutively admitted with SARS-CoV2 confirmed infection. Demographic characteristics, comorbidities, clinical symptoms, laboratory results and radiological tests are described. Results: The study included 255 patients, with a mean age of 70 years; $54.9 \%$ were males. Most common comorbidities were high blood pressure (58\%), dyslipidemia (42.4\%), diabetes (25.5\%) and obesity (24.3\%). Median number of days from the onset of clinical symptoms prior to hospital admission was seven. Most common manifestations prior to admission were fever (74.5\%), dry cough (61.2\%), malaise (51.8\%) and dyspnea (51.0\%); 19 patients (7.4\%) were admitted to the intensive care unit, where mortality was $50 \%$; overall mortality was $16.9 \%$. Conclusions: Our cohort reflects similar characteristics to those of other European series. Mortality was lower than that in similar studies.

KEY WORDS: COVID-19. SARS-CoV-2. Viral pneumonia. Cohort study. Coronavirus. 


\section{Introducción}

En diciembre de 2019, las autoridades sanitarias de China informaron de una serie de casos de pacientes con neumonía cuyo agente causal se identificó posteriormente como SARS-CoV-2 (severe acute respiratory syndrome coronavirus 2). Este virus ha dado lugar a la pandemia actual de COVID-19, declarada por la Organización Mundial de la Salud en marzo de 2020.'

En España, el primer paciente con COVID-19 se registró el 31 de enero de 2020 en Canarias, ${ }^{2}$ pero no fue hasta el 25 de febrero cuando se diagnosticó el primer caso en el Hospital Universitario de La Plana, Castellón, España, importado desde Italia; fue el primero en la Comunidad Valenciana y, en ese momento, había menos de 10 casos diagnosticados en España. ${ }^{3}$ En Europa, los países con más casos notificados son Rusia, Reino Unido, España e Italia. En España, para el 14 de junio se habían notificado 243605 casos confirmados con infección por SARS-CoV-2, de los cuales 124631 precisaron hospitalización, 11619 requirieron ingreso en la Unidad de Cuidados Intensivos (UCl) y 27136 habían fallecido. ${ }^{2}$

El impacto de la pandemia de COVID-19 ha producido la saturación de los sistemas de asistencia sanitaria en prácticamente todos los hospitales de la red pública y ha obligado a reorganizar todos los servicios clínicos. Además, ha sido necesario utilizar los recursos de hospitales privados y otros sistemas de apoyo a la asistencia sanitaria como hoteles medicalizados, hospitales de campaña e, incluso, centros deportivos. ${ }^{4}$

El presente estudio describe las características demográficas, comorbilidades, síntomas clínicos, resultados de laboratorio y pruebas radiológicas de pacientes con infección por SARS-CoV-2 en el momento del ingreso al Hospital Universitario de La Plana, Castellón, España.

\section{Métodos}

Estudio retrospectivo observacional de cohortes realizado en el Hospital Universitario de La Plana, cuyo Departamento de Salud atiende a 187258 habitantes y dispone de 258 camas de hospitalización, entre ellas 12 en la $\mathrm{UCl}$.

Se incluyeron pacientes de 18 años o más ingresados consecutivamente del 26 de febrero al 20 de mayo de 2020, con infección confirmada por SARSCoV-2 mediante prueba diagnóstica de reacción en cadena de la polimerasa (PCR) en tiempo real en una muestra de aspirado nasofaríngeo o esputo. Debido a los posibles resultados falsos negativos, se realizó una segunda prueba diagnóstica si había alta sospecha clínica y radiológica de infección. No se incluyeron los pacientes con infección por SARS-CoV-2 que acudieron a urgencias y que fueron dados de alta a su domicilio. Se excluyeron los pacientes que denegaron su consentimiento informado para recibir tratamiento de la infección por SARS-CoV-2, así como quienes reingresaron y las mujeres embarazadas 0 en periodo de lactancia.

Los datos demográficos, comorbilidades, síntomas clínicos al ingreso, resultados de laboratorio y pruebas radiológicas fueron obtenidos de la historia clínica electrónica.

Los datos analíticos fueron descargados automáticamente desde el programa Cointec Gestlab ${ }^{\circledR} 2020$ (v2.19.4.1493) y las constantes de tensión arterial, temperatura, frecuencia cardiaca y respiratoria del programa Ingresa2, diseñado por la Unidad de Informática del hospital.

Se programó un formulario en la historia clínica electrónica para la recolección segura de los datos que no se descargaban automáticamente de los sistemas de información.

Los datos de carácter personal fueron disociados de la identidad de los pacientes para su posterior análisis estadístico. Para obtener los datos de supervivencia se realizó seguimiento hasta el alta hospitalaria, el traslado a otro hospital o el fallecimiento.

Las variables cuantitativas se expresan mediante medianas y rango intercuartílico (RIC). Las variables categóricas se representan como frecuencias absolutas y porcentajes.

Para el análisis de diferencias de medias se utilizó la prueba $t$ de Student para las variables con distribución normal y la prueba de Mann-Whitney para las variables con distribución no normal.

Para contrastar las variables categóricas se utilizó la prueba de $\chi^{2}$ de Pearson o la prueba exacta de Fisher $y$, en caso necesario, la prueba de tendencia de Mantel-Haenszel. El análisis y tratamiento estadístico de los datos se realizó mediante el programa estadístico SPSS para Windows, versión 19.0. El nivel de significación estadística adoptado para todas las pruebas de contraste fue $p<0.05$.

El protocolo del estudio fue aprobado por el Comité Ético de Investigación con Medicamentos previo a su realización. Debido al diseño observacional retrospectivo de la investigación se obtuvo la 
exención de solicitar el consentimiento informado a los pacientes.

\section{Resultados}

El estudio incluyó 255 pacientes, $54.9 \%$ del sexo masculino, la mediana de edad fue 70 años, variable que se asoció significativamente $(p<0.01)$ con fallecer durante el ingreso; $52.8 \%$ de los pacientes tenía más de 70 años, grupo en el que ocurrieron más fallecimientos (Figura 1). Fallecieron 43 pacientes de los 255 ingresados por COVID-19, que representaron una mortalidad total de nuestra cohorte de $16.9 \%$.

Las comorbilidades más frecuentes fueron hipertensión arterial (HTA, $58 \%$ ), dislipemia (42.4\%), diabetes $(25.5 \%)$ y obesidad (24.3\%) (Tabla 1). Las enfermedades pulmonares (incluidas asma, enfermedad pulmonar obstructiva crónica y otras patologías respiratorias crónicas) estuvieron presentes en 54 pacientes $(21.2 \%)$, sin que fueron predictoras de fallecimiento. La HTA, diabetes, enfermedad cardiovascular, enfermedad renal crónica, enfermedad cerebrovascular y demencia fueron significativamente más frecuentes en los pacientes que fallecieron que en quienes sobrevivieron (Tabla 1). Los pacientes en tratamiento domiciliario con anticoagulación oral fallecieron con más frecuencia que quienes no lo recibían ( $p=0.04)$.

El $12.2 \%$ de los pacientes no presentó ninguna comorbilidad de las registradas (Tabla 1), mientras que 131 pacientes $(51.4 \%)$ presentaron tres o más. Según el índice de comorbilidad de Charlson (versión abreviada), la mediana de la puntuación fue 1 (RIC $=0-2) ; 16.5 \%$ de los pacientes $(n=42)$ presentó elevada comorbilidad (puntuación $\geq 3$ puntos); $16.9 \%$ de los pacientes que fallecieron pertenecieron al grupo de alta comorbilidad en comparación con $13.2 \%$ de los que sobrevivieron. Se presentó una tendencia significativa global $(p=0.005)$ entre los grupos con baja, media y alta comorbilidad.

La mediana del tiempo desde el inicio de síntomas clínicos antes del ingreso fue de siete días $(\mathrm{RIC}=$ 3-9). Las manifestaciones clínicas más frecuentes fueron fiebre $(74.5 \%)$, tos seca $(61.2 \%)$, malestar general $(51.8 \%)$ y disnea $(51.0 \%)$, como se muestra en la Tabla 2. Sin embargo, otros síntomas, como anosmia, ageusia, dolor torácico y dolor de garganta fueron poco frecuentes (menos de $10 \%$ ). La disnea se asoció a fallecimiento, de forma estadísticamente significativa $(p=0.007)$.

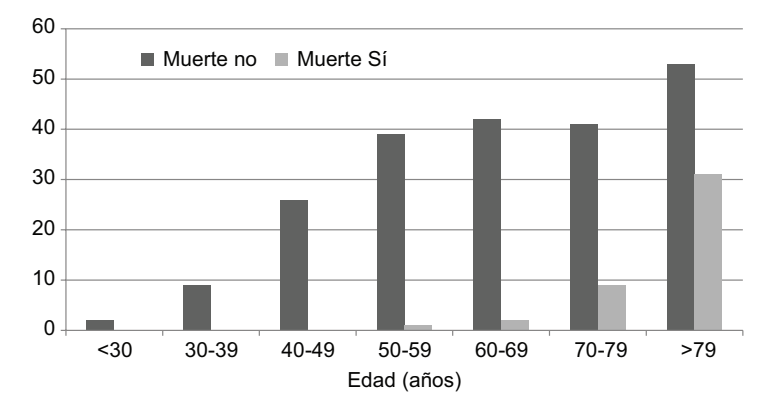

Figura 1. Pacientes por grupos de edad estratificados en función de si se produjo la muerte.

Se observó que $86.6 \%$ de los pacientes presentó imágenes radiológicas de tórax sugestivas de anomalías; el hallazgo más frecuente fue opacidad en vidrio deslustrado (Tabla 3). No se detectaron diferencias significativas en el patrón radiológico entre pacientes fallecidos y supervivientes $(p=0.57)$. La situación respiratoria al ingreso fue buena, con una mediana de SaFi de 442.9 (RIC = 330.4-457.1), pero se encontraron diferencias significativas entre quienes fallecieron $($ mediana $=317.9$, RIC $=148.0-395.2)$ y los supervivientes (mediana $=447.6, \mathrm{RIC}=360.0-457.1$ ), $p<0.01 ; 6.3 \%$ de los pacientes $(n=16)$ presentó un valor de $\mathrm{SaFi} \leq 300$ y requirió ventilación mecánica no invasiva y $9.1 \%(n=23)$ tuvo $\mathrm{SaFi} \leq 200$ y requirió ventilación mecánica invasiva.

En la Tabla 4 se describen los principales resultados de laboratorio que presentaron los pacientes al ingreso hospitalario. Las condiciones patológicas relacionadas con el fallecimiento de los pacientes fueron anemia (hemoglobina $<10 \mathrm{~g} / \mathrm{dL}$ ), elevación del dímero $\mathrm{D}(>1000 \mu \mathrm{g} / \mathrm{mL}), \mathrm{PCR}>10 \mathrm{mg} / \mathrm{dL}$, valores elevados de procalcitonina ( $>0.5 \mathrm{ng} / \mathrm{mL}$ ) y troponina (> $14 \mathrm{pg} / \mathrm{mL}$ ) e insuficiencia renal (filtración glomerular $<59 \mathrm{~mL} /$ minuto $\left./ 1.73 \mathrm{~m}^{2}\right)$. Se presentó anemia de forma más frecuente $(p=0.04)$ en pacientes que fallecieron $(23.8 \%)$ que en los que no fallecieron (11.8\%). Al ingreso, $75 \%$ de los casos presentaron niveles elevados de ferritina (>500 ng/mL) y $62.4 \%$, elevación de dímero $D$ (> $500 \mathrm{ng} / \mathrm{mL}$ ); las diferencias fueron estadísticamente significativas en los valores de este entre el grupo de fallecidos y vivos $(90.6 \%$ versus $72.4 \%, p=0.03)$. La PCR (61.9 versus $32.1 \%$ ) y la procalcitonina (46.7 \% versus 17.6$)$ elevadas al ingreso hospitalario se relacionaron significativamente con la mortalidad, al igual que sucedió con la elevación de la troponina T $(94.7 \%$ versus $40.6 \%)$ y la insuficiencia renal $(73.8 \%$ versus $23.3 \%)$. 
Tabla 1. Características demográficas y comorbilidades de los pacientes con COVID-19 estratificadas en función del resultado de muerte

\begin{tabular}{|c|c|c|c|c|c|c|c|}
\hline & \multicolumn{2}{|c|}{ Total $(n=255)$} & \multicolumn{2}{|c|}{ Muerte sí $(n=43)$} & \multicolumn{2}{|c|}{ Muerte no $(n=212)$} & p \\
\hline \multirow[t]{2}{*}{ Edad en años (mediana y rango) } & \multicolumn{2}{|c|}{$70.0(59.0-82.1)$} & \multicolumn{2}{|c|}{$82.1(78.0-87.4)$} & \multicolumn{2}{|c|}{$66.8(54.0-79.2)$} & $<0.01$ \\
\hline & $\mathrm{n}$ & $\%$ & $\mathrm{n}$ & $\%$ & $\mathrm{n}$ & $\%$ & \\
\hline $\begin{array}{l}\text { Sexo } \\
\text { Hombres } \\
\text { Mujeres }\end{array}$ & $\begin{array}{l}140 \\
115\end{array}$ & $\begin{array}{l}54.9 \\
45.1\end{array}$ & $\begin{array}{l}19 \\
24\end{array}$ & $\begin{array}{l}44.2 \\
55.8\end{array}$ & $\begin{array}{c}121 \\
91\end{array}$ & $\begin{array}{l}57.1 \\
42.9\end{array}$ & 0.12 \\
\hline HTA & 148 & 58.0 & 35 & 81.4 & 113 & 53.3 & $<0.01$ \\
\hline Diabetes & 65 & 25.5 & 18 & 41.9 & 47 & 22.2 & $<0.01$ \\
\hline Cardiopatía isquémica & 23 & 9.0 & 5 & 11.6 & 18 & 8.5 & 0.51 \\
\hline Enfermedad renal crónica & 49 & 19.2 & 20 & 46.5 & 29 & 13.7 & $<0.01$ \\
\hline EPOC & 21 & 8.2 & 5 & 11.6 & 16 & 7.5 & 0.38 \\
\hline Asma & 19 & 7.5 & 6 & 14.0 & 13 & 6.1 & 0.08 \\
\hline Otras enfermedades pulmonares crónicas & 24 & 9.4 & 6 & 14.0 & 18 & 8.5 & 0.26 \\
\hline Insuficiencia cardiaca & 21 & 8.2 & 5 & 11.6 & 16 & 7.5 & 0.38 \\
\hline Cirrosis & 4 & 1.6 & 1 & 2.3 & 3 & 1.4 & 0.66 \\
\hline Neoplasia & 31 & 12.2 & 8 & 18.6 & 23 & 10.8 & 0.16 \\
\hline Enfermedad cardiovascular & 71 & 27.8 & 18 & 41.9 & 53 & 25 & 0.03 \\
\hline Enfermedad cerebrovascular & 18 & 7.1 & 7 & 16.3 & 11 & 5.2 & 0.01 \\
\hline Dislipemia & 108 & 42.4 & 17 & 39.5 & 91 & 42.9 & 0.68 \\
\hline $\begin{array}{l}\text { Tabaquismo } \\
\text { Nunca ha fumado } \\
\text { Fumador } \\
\text { Exfumador }\end{array}$ & $\begin{array}{l}192 \\
23 \\
40\end{array}$ & $\begin{array}{c}75.3 \\
9 \\
15.7\end{array}$ & $\begin{array}{c}37 \\
1 \\
5\end{array}$ & $\begin{array}{c}86 \\
4.3 \\
11.6\end{array}$ & $\begin{array}{c}155 \\
22 \\
35\end{array}$ & $\begin{array}{l}73.1 \\
10.4 \\
16.5\end{array}$ & $0.14^{\dagger}$ \\
\hline Obesidad $\left(\mathrm{IMC} \geq 30 \mathrm{~kg} / \mathrm{m}^{2}\right)$ & 62 & 24.3 & 11 & 25.6 & 51 & 24.1 & 0.83 \\
\hline VIH & 4 & 1.6 & 1 & 2.3 & 3 & 1.4 & 0.66 \\
\hline Enfermedad inflamatoria intestinal & 6 & 2.4 & 1 & 2.3 & 5 & 2.4 & 0.99 \\
\hline Enfermedades autoinmunes & 15 & 5.9 & 3 & 7 & 12 & 5.7 & 0.74 \\
\hline Demencia & 27 & 10.6 & 10 & 23.3 & 17 & 8.0 & $<0.01$ \\
\hline Embolia pulmonar o trombosis venosa profunda & 4 & 1.6 & 0 & 0.0 & 4 & 1.9 & 0.36 \\
\hline Artritis reumatoide & 6 & 2.4 & 2 & 4.7 & 4 & 1.9 & 0.28 \\
\hline $\begin{array}{l}\text { Tratamiento domiciliario } \\
\text { Anticoagulante } \\
\text { IECA o ARA II } \\
\text { Fármacos biológicos }\end{array}$ & $\begin{array}{c}30 \\
115 \\
2\end{array}$ & $\begin{array}{c}11.8 \\
45.1 \\
0.8\end{array}$ & $\begin{array}{c}9 \\
21 \\
0\end{array}$ & $\begin{array}{c}20.9 \\
48.8 \\
0.0\end{array}$ & $\begin{array}{c}21 \\
94 \\
2\end{array}$ & $\begin{array}{c}9.9 \\
44.3 \\
0.9\end{array}$ & $\begin{array}{l}0.04 \\
0.59 \\
0.69\end{array}$ \\
\hline
\end{tabular}

HTA = hipertensión arterial, EPOC = enfermedad pulmonar obstructiva crónica, IMC = índice de masa corporal, VIH = virus de la inmunodeficiencia humana, IECA = inhibidor de la enzima convertidora de angiotensina, ARA II = bloqueador de receptores de la angiotensina II. tPrueba de tendencia de Mantel-Haenszel.

Casi la mitad de los pacientes $(47.4 \%)$ presentaba linfopenia al ingreso (recuento de linfocitos $<1.0 \times$ $10 \%$ L), mientras que $29.2 \%$ tenía trombocitopenia (plaquetas < 150000 ), sin que estos trastornos estuvieran relacionados con mortalidad.
No se encontró relación significativa de la mortalidad con niveles elevados de lactado deshidrogenasa, creatinfosfoquinasa, albuminemia ni alargamiento del tiempo de protrombina o de tromboplastina parcial activada. 
Tabla 2. Signos y síntomas que presentaban los pacientes al ingreso

\begin{tabular}{|c|c|c|c|c|c|c|c|}
\hline \multirow[t]{2}{*}{$\begin{array}{l}\text { Signo y } \\
\text { o síntoma }\end{array}$} & \multicolumn{2}{|c|}{$\begin{array}{c}\text { Total } \\
(\mathrm{n}=255)\end{array}$} & \multicolumn{2}{|c|}{$\begin{array}{c}\text { Muerte sí } \\
(n=43)\end{array}$} & \multicolumn{2}{|c|}{$\begin{array}{c}\text { Muerte no } \\
(\mathrm{n}=212)\end{array}$} & \multirow[t]{2}{*}{ p } \\
\hline & $\mathrm{n}$ & $\%$ & $n$ & $\%$ & $\mathrm{n}$ & $\%$ & \\
\hline Fiebre & 190 & 74.5 & 32 & 74.4 & 158 & 74.5 & 0.99 \\
\hline Disnea & 130 & 51.0 & 30 & 69.8 & 100 & 47.2 & $<0.01$ \\
\hline Tos seca & 156 & 61.2 & 23 & 53.5 & 133 & 62.7 & 0.26 \\
\hline Expectoración & 36 & 14.1 & 7 & 16.3 & 29 & 13.7 & 0.66 \\
\hline Dolor de garganta & 19 & 7.5 & 1 & 2.3 & 18 & 8.5 & 0.21 \\
\hline Mialgias & 53 & 20.8 & 7 & 16.3 & 46 & 21.7 & 0.43 \\
\hline Cefalea & 28 & 11.0 & 1 & 2.3 & 27 & 12.7 & 0.06 \\
\hline Mareo & 19 & 7.5 & 2 & 4.7 & 17 & 8.0 & 0.75 \\
\hline Diarrea & 58 & 22.7 & 11 & 25.6 & 47 & 22.2 & 0.63 \\
\hline Dolor torácico & 18 & 7.1 & 1 & 2.3 & 17 & 8.0 & 0.32 \\
\hline Malestar general & 132 & 51.8 & 21 & 48.8 & 111 & 52.4 & 0.67 \\
\hline Anosmia & 18 & 7.1 & 0 & 0.0 & 18 & 8.5 & 0.05 \\
\hline Ageusia & 19 & 7.5 & 1 & 2.3 & 18 & 8.5 & 0.21 \\
\hline
\end{tabular}

Tabla 3. Resultados de la radiografía de tórax

\begin{tabular}{|l|c|c|c|c|c|c|c|}
\hline Resultado & \multicolumn{2}{|c|}{$\begin{array}{c}\text { Total } \\
(\mathrm{n=255})\end{array}$} & \multicolumn{2}{|c|}{$\begin{array}{c}\text { Muerte sí } \\
(\mathrm{n}=\mathbf{4 3})\end{array}$} & \multicolumn{2}{|c|}{$\begin{array}{c}\text { Muerte no } \\
(\mathrm{n}=\mathbf{2 1 2})\end{array}$} & $\mathrm{p}$ \\
\cline { 2 - 9 } & $\mathrm{n}$ & $\%$ & $\mathrm{n}$ & $\%$ & $\mathrm{n}$ & $\%$ & \\
\hline Normal & 34 & 13.4 & 4 & 9.3 & 30 & 14.2 & $0.57^{\dagger}$ \\
\hline Opacidades & 130 & 51.0 & 22 & 51.2 & 108 & 50.9 & \\
\hline Patrón intersticial & 80 & 31.4 & 16 & 37.2 & 64 & 30.2 & \\
\hline $\begin{array}{l}\text { Lesiones no } \\
\text { COVID-19 }\end{array}$ & 11 & 4.3 & 1 & 2.3 & 10 & 4.7 & \\
\hline
\end{tabular}

tPrueba de tendencia de Mantel-Haenszel.

Al aplicar la escala CURB-65 al ingreso hospitalario, 34 pacientes (13.5\%) presentaban riesgo severo y, por lo tanto, requerían ingreso en la $\mathrm{UCl} ; 28.7 \%$ necesitaba ingreso hospitalario por riesgo medio y $56.9 \%$ no precisaba ingreso. Nueve pacientes ingresaron directamente a la $\mathrm{UCl}$ desde el Servicio de Urgencias; la mediana de la duración de la estancia hospitalaria fue de ocho días ( $\mathrm{RIC}=5-15$ ), si bien en los pacientes que sobrevivieron fue mayor (nueve días, $\mathrm{RIC}=6-17$ ) en comparación con quienes fallecieron (cinco días, $\mathrm{RIC}=2.5-9.5$ ), sin diferencias significativas $(p=0.08)$.
Diecinueve pacientes $(7.4 \%)$ requirieron ingreso a la $\mathrm{UCl}$, de los cuales nueve fueron trasladados a otros hospitales. La mediana de la estancia en la UCI fue de 34 días ( $R I C=26-45)$, sin diferencias significativas $(p=0.66)$ entre los que fallecieron y quienes sobrevivieron: 32 ( $R I C=26-42)$ y 36 días $(R I C=30-45)$, respectivamente. La mortalidad de los pacientes que ingresaron en la $\mathrm{UCl}$ fue de $50 \%$ y los pacientes tuvieron cinco veces más riesgo de fallecer cuando ingresaban en esta unidad (RM $=5.4$, IC $95 \%=1.5$ 19.7, $p<0.01$ ).

\section{Discusión}

Se han observado diferencias sustanciales entre cohortes de COVID-19 de países asiáticos ${ }^{5-10}$ y occidentales. ${ }^{11-16}$ En general, los estudios publicados en China incluyeron pacientes más jóvenes, con edad entre 47 y 56 años, ${ }^{5,8}$ a diferencia de los pacientes de nuestro estudio y el resto de las cohortes occidentales, ${ }^{11-16}$ en quienes la edad es superior a los 60 años. Sin embargo, no hubo diferencias en la proporción de varones y mujeres en los diferentes estudios.

Nuestros pacientes presentaron múltiples comorbilidades: HTA, dislipemia y diabetes, entre las más frecuentes, al igual que los de otras series publicadas anteriormente. ${ }^{11-16}$ Estos hallazgos no fueron comunicados en los estudios iniciales. Así, en la serie de Guan et al..$^{5}$ solamente $15 \%$ tenía HTA y $7.4 \%$, diabetes; en otras series chinas ${ }^{8,10}$ se registró $30 \%$ de pacientes con HTA y $10 \%$ con diabetes. Estas diferencias pueden deberse a que se trata de una población más joven y con menor comorbilidad.

Por otra parte, la prevalencia de HTA en la población española ${ }^{17}$ es de $42.6 \%$, menor a la que se observó en nuestros pacientes $(58 \%)$. Posiblemente existe mayor susceptibilidad a infectarse por SARS-CoV-2 debido a una sobreexpresión de la enzima convertidora de la angiotensina 2 en estos pacientes. ${ }^{18}$ Encontramos menor prevalencia de obesidad en nuestros pacientes $(23.4 \%)$ que la comunicada por Richardson et al. ${ }^{11}$ en Nueva York (41.7\%), debido a la distinta prevalencia de esta enfermedad en la población norteamericana. Las otras comorbilidades estudiadas fueron menos frecuentes: la prevalencia de $8.2 \%$ de enfermedad pulmonar obstructiva crónica en nuestra cohorte fue inferior a $10.2 \%$ identificado en la población española. ${ }^{19}$ Como se muestra en la Tabla 1 , los principales factores al ingreso predictores de mortalidad fueron edad, HTA, diabetes, enfermedad renal crónica, demencia, enfermedad cardiovascular y 
Tabla 4. Resultados de laboratorio de los pacientes con COVID 19 al ingreso hospitalario

\begin{tabular}{|c|c|c|c|c|c|c|}
\hline \multirow[t]{2}{*}{ Prueba de Laboratorio, } & $\begin{array}{l}\text { Pruebas } \\
\text { realizadas }\end{array}$ & Total & Muerte sí $(n=43)$ & $\begin{array}{l}\text { Muerte no } \\
(\mathrm{n}=212)\end{array}$ & \multirow[t]{2}{*}{$p$} & \multirow[t]{2}{*}{$\begin{array}{l}\text { Rango de } \\
\text { referencia* }\end{array}$} \\
\hline & $\mathrm{n}$ & Mediana (RIC) & Mediana (RIC) & Mediana (RIC) & & \\
\hline $\begin{array}{l}\text { Leucocitos } \\
\text { (recuento absoluto } \times 10^{9} / \mathrm{L} \text { ) }\end{array}$ & 255 & $\begin{array}{c}6.3 \\
(5.0-8.7)\end{array}$ & $\begin{array}{c}6,8 \\
(5.4-9.7)\end{array}$ & $\begin{array}{c}6.2 \\
(4.9-8.7)\end{array}$ & 0.18 & $4.80-11.00$ \\
\hline $\begin{array}{l}\text { Neutrófilos } \\
\text { (recuento absoluto } \times 10^{9} / \mathrm{L} \text { ) }\end{array}$ & 255 & $\begin{array}{c}4.7 \\
(3.3-6.9)\end{array}$ & $\begin{array}{c}5.5 \\
(4.5-7.5)\end{array}$ & $\begin{array}{c}4,6 \\
(3.2-6.8)\end{array}$ & 0.02 & $1.90-8.00$ \\
\hline $\begin{array}{l}\text { Linfocitos } \\
\left.\text { (recuento } \times 10^{9} / \mathrm{L}\right)\end{array}$ & 255 & $\begin{array}{c}1.0 \\
(0.7-1.4)\end{array}$ & $\begin{array}{c}0.8 \\
(0.5-1.2)\end{array}$ & $\begin{array}{c}1,0 \\
(0.7-1.4)\end{array}$ & 0.03 & $0.90-4.50$ \\
\hline $\begin{array}{l}\text { Plaquetas } \\
\text { (recuento } \times 10^{9} / \mathrm{L} \text { ) }\end{array}$ & 255 & $\begin{array}{c}183 \\
(143-235)\end{array}$ & $\begin{array}{c}174 \\
(145-212)\end{array}$ & $\begin{array}{c}186 \\
(143-236)\end{array}$ & 0.13 & $130-400$ \\
\hline Hemoglobina (g/dL) & 255 & $\begin{array}{c}13.8 \\
(12.8-14.9)\end{array}$ & $\begin{array}{c}12.9 \\
(12.2-14.4)\end{array}$ & $\begin{array}{c}13.9 \\
(12.9-15.0)\end{array}$ & 0.02 & $12.0-18.0$ \\
\hline D-dimero $(\mu \mathrm{g} / \mathrm{mL})$ & 236 & $\begin{array}{c}762.0 \\
(497.5-1321.5)\end{array}$ & $\begin{array}{c}1222,5 \\
(806.0-1894.0)\end{array}$ & $\begin{array}{c}701,0 \\
(473.5-1241.5)\end{array}$ & 0.23 & $0-500$ \\
\hline $\begin{array}{l}\text { Tiempo de } \\
\text { protrombina (segundos) }\end{array}$ & 250 & $\begin{array}{c}13.1 \\
(12.1-14.2)\end{array}$ & $\begin{array}{c}12,8 \\
(12.1-15.1)\end{array}$ & $\begin{array}{c}13,2 \\
(12.1-14.2)\end{array}$ & 0.48 & $9-13$ \\
\hline $\begin{array}{l}\text { Tiempo parcial de tromboplastina } \\
\text { activada (segundos) }\end{array}$ & 220 & $\begin{array}{c}30.35 \\
(28.50-33.30)\end{array}$ & $\begin{array}{c}31,00 \\
(29.40-36.60)\end{array}$ & $\begin{array}{c}30,20 \\
(28.20-32.90)\end{array}$ & 0.40 & $20-40$ \\
\hline Fibrinógeno (mg/dL) & 9 & $\begin{array}{c}662.5 \\
(578.0-880.0)\end{array}$ & NA & $\begin{array}{c}662,5 \\
(578.0-880.0)\end{array}$ & NA & $200-400$ \\
\hline Glucemia (mg/dL) & 253 & $\begin{array}{c}121.0 \\
(106.0-143.0)\end{array}$ & $\begin{array}{c}134,5 \\
(118.0-200.0)\end{array}$ & $\begin{array}{c}118,0 \\
(106.0-145.5)\end{array}$ & 0.03 & $82-115$ \\
\hline Creatinina sérica (mg/dL) & 252 & $\begin{array}{c}0.95 \\
(0.75-1.23)\end{array}$ & $\begin{array}{c}1,24 \\
(0.99-1.73)\end{array}$ & $\begin{array}{c}0,91 \\
(0.73-1.14)\end{array}$ & $<0.01$ & $0.70-1.20$ \\
\hline Urea sérica (mg/dL) & 253 & $\begin{array}{c}38.0 \\
(28.0-57.0)\end{array}$ & $\begin{array}{c}58,0 \\
(40.0-99.0)\end{array}$ & $\begin{array}{c}36,0 \\
(27.0-51.5)\end{array}$ & $<0.01$ & $10-71$ \\
\hline $\begin{array}{l}\text { Filtración glomerular (CKD-EPI, } \\
\mathrm{mL} / \mathrm{min} / 1,73 \mathrm{~m}^{2} \text { ) }\end{array}$ & 252 & $\begin{array}{c}77.42 \\
(51.42-93.70)\end{array}$ & $\begin{array}{c}44,03 \\
(31.36-59.31)\end{array}$ & $\begin{array}{c}82,35 \\
(59.75-97.32)\end{array}$ & $<0.01$ & $\begin{array}{l}\mathrm{FG}^{\circ}>\circ 90 \\
(\text { normal) }\end{array}$ \\
\hline Sodio ión (mmol/L) & 250 & $\begin{array}{c}138.95 \\
(136.50-141.20)\end{array}$ & $\begin{array}{c}138,05 \\
(136.20-141.40)\end{array}$ & $\begin{array}{c}139,10 \\
(136.55-141.15)\end{array}$ & 0.78 & $136-146$ \\
\hline Potasio ión (mmol/L) & 224 & $\begin{array}{c}4.24 \\
(3.88-4.48)\end{array}$ & $\begin{array}{c}4.33 \\
(3.92-4.63)\end{array}$ & $\begin{array}{c}4.22 \\
(3.88-4.44)\end{array}$ & 0.06 & $3.5-5.1$ \\
\hline Fosfato (mg/dL) & 113 & $\begin{array}{c}2.91 \\
(2.53-3.38)\end{array}$ & $\begin{array}{c}3,10 \\
(2.72-4.55)\end{array}$ & $\begin{array}{c}2.90 \\
(2.49-3.32)\end{array}$ & 0,04 & $2.7-4.5$ \\
\hline Calcio total (mg/dL) & 111 & $\begin{array}{c}9.20 \\
(8.75-9.50)\end{array}$ & $\begin{array}{c}9,40 \\
(8.80-9.90)\end{array}$ & $\begin{array}{c}9.20 \\
(8.75-9.45)\end{array}$ & 0.35 & $8.8-10.2$ \\
\hline Albúmina (g/dL) & 113 & $\begin{array}{c}3.87 \\
(3.41-4.11)\end{array}$ & $\begin{array}{c}3.36 \\
(3.04-3.97)\end{array}$ & $\begin{array}{c}3.91 \\
(3.55-4.12)\end{array}$ & $<0.01$ & $3.97-4.94$ \\
\hline Bilirubina total (mg/dL) & 123 & $\begin{array}{c}0.52 \\
(0.34-0.65)\end{array}$ & $\begin{array}{c}0.56 \\
(0.45-0.77)\end{array}$ & $\begin{array}{c}0.51 \\
(0.42-0.63)\end{array}$ & 0.07 & $0.1-1.2$ \\
\hline $\begin{array}{l}\text { Aspartato aminotransferasa } \\
(\mathrm{UI} / \mathrm{L})\end{array}$ & 60 & $\begin{array}{c}63.4 \\
(49.7-95.9)\end{array}$ & $\begin{array}{c}65.4 \\
(54.6-106.3)\end{array}$ & $\begin{array}{c}64.8 \\
(49.2-95.9)\end{array}$ & 0.85 & $5-40$ \\
\hline $\begin{array}{l}\text { Alanina aminotransferasa } \\
\text { (UI/L) }\end{array}$ & 222 & $\begin{array}{c}25.2 \\
(16.0-40.5)\end{array}$ & $\begin{array}{c}19,9 \\
(15.4-35.2)\end{array}$ & $\begin{array}{c}26.9 \\
(16.5-42.0)\end{array}$ & 0.29 & $5-41$ \\
\hline Creatinfosfoquinasa total (UI/L) & 171 & $\begin{array}{c}91.0 \\
(55.0-149.5)\end{array}$ & $\begin{array}{c}87.0 \\
(68.0-153.0)\end{array}$ & $\begin{array}{c}91.0 \\
(54.0-146.0)\end{array}$ & 0.29 & $39-308$ \\
\hline
\end{tabular}


Tabla 4. Resultados de laboratorio de los pacientes con COVID 19 al ingreso hospitalario (Continuación)

\begin{tabular}{|c|c|c|c|c|c|c|}
\hline \multirow[t]{2}{*}{ Prueba de Laboratorio, } & $\begin{array}{l}\text { Pruebas } \\
\text { realizadas }\end{array}$ & Total & Muerte sí $(n=43)$ & $\begin{array}{l}\text { Muerte no } \\
(n=212)\end{array}$ & \multirow[t]{2}{*}{ p } & \multirow[t]{2}{*}{$\begin{array}{l}\text { Rango de } \\
\text { referencia* }\end{array}$} \\
\hline & $\mathrm{n}$ & Mediana (RIC) & Mediana (RIC) & Mediana (RIC) & & \\
\hline Deshidrogensas láctica (UI/L) & 218 & $\begin{array}{c}284.0 \\
(221.0-397.5)\end{array}$ & $\begin{array}{c}349.0 \\
(279.5-483.0)\end{array}$ & $\begin{array}{c}277.5 \\
(220.0-380.0)\end{array}$ & 0.09 & $135-225$ \\
\hline PCR (mg/dL) & 251 & $\begin{array}{c}7.32 \\
(2.97-12.8)\end{array}$ & $\begin{array}{c}12.47 \\
(8.45-21.19)\end{array}$ & $\begin{array}{c}5.59 \\
(2.44-11.78)\end{array}$ & $<0.01$ & $0.1-1.0$ \\
\hline Procalcitonina (ng/mL) & 115 & $\begin{array}{c}0.100 \\
(0.080-0.330)\end{array}$ & $\begin{array}{c}0.388 \\
(0.164-1.125)\end{array}$ & $\begin{array}{c}0.118 \\
(0.075-0.270)\end{array}$ & 0.68 & $0.0-0.5$ \\
\hline Ferritina (ng/mL) & 175 & $\begin{array}{c}741.5 \\
(337.9-1440.4)\end{array}$ & $\begin{array}{c}1278.8 \\
(561.2-1757.2)\end{array}$ & $\begin{array}{c}724.2 \\
(313.2-1242.2)\end{array}$ & 0.25 & $30-400$ \\
\hline Troponina T (pg/mL) & 117 & $\begin{array}{c}13.98 \\
(6.84-29.73)\end{array}$ & $\begin{array}{c}41.13 \\
(24.52-109.50)\end{array}$ & $\begin{array}{c}12.64 \\
(6.23-23.66)\end{array}$ & 0.06 & $\begin{array}{l}\text { Límite superior: } \\
\qquad 14\end{array}$ \\
\hline Interleuquina-6 (pg/mL) & 9 & $\begin{array}{c}25.70 \\
(17.34-75.53)\end{array}$ & $\begin{array}{c}131.27 \\
(39.63-487.25)\end{array}$ & $\begin{array}{c}24.83 \\
(17.34-25.70)\end{array}$ & 0.27 & $0-8$ \\
\hline
\end{tabular}

*Proporcionado por el Servicio de Laboratorio Clínico del Hospital Universitario de La Plana. $\mathrm{RIC}=$ rango intercuartílico, $\mathrm{NA}=$ no evaluable, $\mathrm{PCR}=$ proteína $\mathrm{C}$ reactiva .

cerebrovascular, al igual que en otro estudio en población española. Borobia et al. ${ }^{16}$ encontraron, además, mayor mortalidad en pacientes con enfermedad pulmonar obstructiva crónica, enfermedad reumatológica y neoplasias previas.

Los síntomas más frecuentes al ingreso fueron los que ya se han descrito con anterioridad: ${ }^{5-10}$ fiebre, tos seca, malestar general y disnea, pero solo la disnea al ingreso se asoció con riesgo de fallecer, a diferencia de los hallazgos de Borobia et al., ${ }^{16}$ quienes encontraron más síntomas predictores de mortalidad al ingreso: tos, cefalea, diarrea, mialgias, expectoración, anosmia, náuseas y vómitos.

Encontramos hallazgos patológicos en $86.6 \%$ de los estudios radiológicos obtenidos al ingreso, prevalencia superior a la de otras series, ${ }^{5,10}$ lo que puede indicar una evolución más avanzada de la enfermedad posiblemente porque los pacientes acudieron más tarde al hospital. En efecto, nuestros pacientes tuvieron síntomas clínicos siete días antes del ingreso.

En nuestra serie, de forma similar a otros estudios, ${ }^{5,8,11}$ observamos linfopenia, albuminemia y valores elevados de dímero $D$, lactado deshidrogenasa, PCR y ferritina sérica, lo que concuerda con un perfil proinflamatorio al ingreso de los pacientes. El $7.4 \%$ $(n=19)$ de los pacientes ingresó a la $\mathrm{UCl}$, de los cuales nueve tuvieron que ser trasladados a la $\mathrm{UCl}$ de otros hospitales, número inferior al reportado en otras series $8,11,13,15$ en las que se alcanzaron valores de 14.2 a $30 \%$.
Fallecieron 43 pacientes de nuestra cohorte, que representaron una mortalidad global de $16.8 \%$; la prevalencia de fallecimientos fue más elevada en los pacientes que ingresaron a la $\mathrm{UCI}(50 \%)$, al igual que se informa en otras series. ${ }^{11}$ Si hubiésemos incluido dos pacientes fallecidos de los trasladados a la UCl de otros hospitales, la mortalidad sería de $17.6 \%$, aunque seguiría siendo inferior a $21.2 \%$ señalado por Richardson ${ }^{11}$ o $26 \%$ registrado en la cohorte ISARIC. ${ }^{15}$ En otra serie española también se registró menor mortalidad $(20.7 \%),{ }^{16}$ como en nuestra investigación. Como se observa en la Figura 1, la mayor mortalidad se concentró en los mayores de 60 años (97.7\%), en forma similar al hallazgo de Borobia $(95.8 \%){ }^{16}$

La mortalidad tanto en Europa como en Estados Unidos es superior a la de China: Guan et al. ${ }^{5}$ comunicaron una mortalidad de $1.4 \%$ y Wang et al., ${ }^{8}$ de $4.3 \%$.

Nuestro estudio no está exento de limitaciones. Al realizarse en un único centro hospitalario podría existir sesgo de distinta práctica clínica, que se ha intentado reducir con el empleo de las recomendaciones de tratamiento aprobadas a nivel nacional. Al tratarse de un estudio retrospectivo, no se pueden extraer relaciones de causalidad respecto a los hallazgos encontrados, que deberán refrendarse con análisis prospectivos.

En conclusión, las características de los pacientes atendidos por COVID-19 en el Hospital Universitario de La Plana, en Castellón, España, reflejan características 
similares a los de otras series europeas, pero diferentes a las registradas en población china o norteamericana. La mortalidad de nuestro estudio fue inferior a la de otras poblaciones similares.

\section{Agradecimientos}

Este artículo forma parte de la tesis doctoral de Laura Álvarez en la Universidad de Granada, España, Programa de Doctorado en Farmacia del Departamento de Farmacia.

\section{Conflicto de intereses}

Los autores declaran no tener ningún conflicto de interés.

\section{Financiamiento}

Los autores no recibieron patrocinio para llevar a cabo este artículo.

\section{Responsabilidades éticas}

Protección de personas y animales. Los autores declaran que para esta investigación no realizaron experimentos en seres humanos ni en animales.

Confidencialidad de los datos. Los autores declaran que siguieron los protocolos de su centro de trabajo sobre la publicación de datos de pacientes.

Derecho a la privacidad y consentimiento informado. Los autores declaran que en este artículo no aparecen datos de pacientes.

\section{Bibliografía}

1. World Health Organizatio [Internet]. Suiza: Statement - preparing for the autumn is a priority now at the WHO Regional Office for Europe; 2020.
2. Ministerio de Sanidad, Consumo y Bienestar Social [Internet]. España: Actualización $n^{\circ}$ 136. Enfermedad por el coronavirus (COVID-19). 14.06.2020; 2020.

3. Generalitat Valenciana [Internet]. España: Información estadística sobre coronavirus en la Comunidad Valenciana - Conselleria de Sanidad Universal y Salud Pública; 2020.

4. Prinja S, Pandav CS. Economics of COVID-19: challenges and the way forward for health policy during and after the pandemic. Indian J Public Health. 2020;64:S231-S233.

5. Guan W, Ni Z, Hu Y, Liang W, Ou C, He J, et al. Clinical characteristics of coronavirus disease 2019 in China. N Engl J Med. 2020;382: $1708-1720$

6. Huang C, Wang Y, Li X, Ren L, Zhao J, Hu Y, et al. Clinical features of patients infected with 2019 novel coronavirus in Wuhan, China. Lancet. 2020;395(10223):497-506.

7. Jiang F, Deng L, Zhang L, Cai Y, Cheung CW, Xia Z, et al: Review of the clinical characteristics of coronavirus disease 2019 (COVID-19). J Gen Intern Med. 2020;35:1545-1549.

8. Wang D, Hu B, Hu C, Zhu F, Liu X, Zhang J, et al. Clinical characteristics of 138 hospitalized patients with 2019 novel coronavirus-infected pneumonia in Wuhan, China. JAMA. 2020;323:1061-1069.

9. Zhang JJ, Dong X, Cao Y, Yuan YD, Yang YB, Yan YQ. Clinical characteristics of 140 patients infected with SARS-CoV-2 in Wuhan, China. Allergy. 2020;75:1730-1741.

10. Zhou F, Yu T, Du R, Fan G, Liu Y, Liu Z et al. Clinical course and risk factors for mortality of adult inpatients with COVID-19 in Wuhan, China: a retrospective cohort study. Lancet. 2020;395(10229):1054-1062.

11. Richardson S, Hirsch JS, Narasimhan M, Crawford JM, McGinn T, Davidson KW, et al. Presenting characteristics, comorbidities, and outcomes among 5700 patients hospitalized with COVID-19 in the New York City Area. JAMA. 2020;323:2052-2059.

12. Argenziano M, Bruce S, Slater C, Tiao J, Baldwin M, Barr R, et al. Characterization and clinical course of 1000 patients with coronavirus disease 2019 in New York: retrospective case series. BMJ. 2020;369:m1996.

13. Myers LC, Parodi SM, Escobar GJ, Liu VX. Characteristics of hospitalized adults with COVID-19 in an integrated health care system in California. JAMA 2020;323(21):2195-2198

14. Grasselli G, Zangrillo A, Zanella A, Antonelli M, Cabrini L, Castelli A, et al. Baseline characteristics and outcomes of 1591 patients infected with SARS-CoV-2 admitted to ICUs of the Lombardy Region, Italy. JAMA. 2020;323:1574-1581.

15. Docherty AB, Harrison EM, Green CA, Hardwick HE, Pius R, Norman L et al. Features of 20133 UK patients in hospital with covid-19 using the ISARIC WHO Clinical Characterization Protocol: prospective observational cohort study. BMJ. 2020;369:m1985.

16. Borba AM, Carcas AJ, Arnalich F, Álvarez-Sala R, Montserrat-Villatoro J, Quintana M, et al. A cohort of patients with COVID-19 in a major teaching hospital in Europe. J Clin Med. 2020;9:1733.

17. Menéndez E, Delgado E, Fernández-Vega F, Prieto MA, Bordiú E, Calle $A$, et al. Prevalencia, diagnóstico, tratamiento y control de la hipertensión arterial en España. Resultados del estudio Di@bet.es. Rev Esp Cardiol. 2016;69:572-578.

18. Díaz JH. Hypothesis: angiotensin-converting enzyme inhibitors and angiotensin receptor blockers may increase the risk of severe COVID-19. J Travel Med. 2020;27:taaa041.

19. Miravitlles M, Soriano JB, García-Río F, Muñoz L, Durán-Tauleria E, Sánchez G, et al. Prevalence of COPD in Spain: impact of undiagnosed COPD on quality of life and daily life activities. Thorax. 2009;64: 863-868. 